\title{
Investigation and Aanlysis of “Zhai” Lifestyle’s Effects on College Students’ Physical and Mental Health
}

\author{
Xiaohua Zhang ${ }^{1, *}$ \\ ${ }^{1}$ School of Business, Sias International University, Henan, China \\ *Correspondence: School of Business, Sias International University, Renmin Road NO.168, Xinzheng 451150, \\ Henan, China. E-mail: rabbit3600@163.com
}

Received: June 26, 2013

Accepted: July 30, 2013

Online Published: August 4, 2013

doi:10.5430/wje.v3n4p26

URL: http://dx.doi.org/10.5430/wje.v3n4p26

\begin{abstract}
With the development of science and technology, especially the popularity of the Internet, mobile phone, and computer, college students' lifestyle is changing, which leads to the "Zhai" phenomenon. More and more students house in the dorm or home. They reduce the communication with the outside world. This phenomenon not only reduces the survivability of the students, but also causes no small impact to their physical and mental health. Students are the hope of the society. If they are indulged in "Zhai" lifestyle, they will be difficult to take the responsibilities of the future development in the country. "Zhai" has become stern problems in current society, schools and families. No doubt a study on the relevant topic is of realistic value. Under the background, based on survey data, I do quantitative analysis combined with qualitative analysis, on the basis of extensive reading relevant literature, trying to use the theory to extract the data. The conclusions are: More and more college students (especially male and senior students) choose "Zhai" lifestyle; The results confirm "Zhai" produces plenty of negative influence on college students' physical and mental health; Although "Zhai” lifestyle has effected students health, they have not aware of the harm of "Zhai". So at last the paper gives the rational proposal.
\end{abstract}

Keywords: "Zhai” lifestyle; physical and mental health; interpersonal communication; spare time

\section{Introduction}

\subsection{The Research Background and Significance}

Recently, the frequent news about the sudden death of college students in the long-distance race and college students' suicide and homicide pushed the public discussion on the current health status of college students. What led to this phenomenon on earth? "Zhai" (that is, "otaku", or indoorsman and indoorswoman) has become a very important factor.

Today, with the development of technology and science, more and more supplementary tools such as mobile phone, email, internet, fetion, micro-channel, MSN, Skype, blog, etc. reduce the distance between people greatly, people can communicate with each other conveniently and swiftly. On the other hand, more people choose to stay at home all day, not willing to go out, face-to-face communications are becoming rare. The phenomenon is significant in the young generation, especially among college students. The so-called "Zhai" come into being. However, this lifestyle brings lots of problems. It is not only a health problem, but also "Zhai” has an impact on college students' mental health. Many college students speak out on the network, while in the real world are poor expression. During the process of interaction with people, psychological and personality problems are becoming increasingly evident. Severe communication barriers, and even fear, depression, suicide, murder are not uncommon at all major colleges and universities due to excessive addiction on the network and dependence on the virtual world. Students are the hope of the society. If they are indulged in "Zhai" lifestyle, they will be difficult to take the responsibilities of the future development in the country. "Zhai" has become stern problems in current society, schools and families. No doubt a study on the relevant topic is of realistic value.

\subsection{Literature Review}

At present there is a little literature about "Zhai" lifestyle, and the effects that "Zhai" has on college students ' 
physical and mental health have been reported rarely. Foreign Scholars Mehdi Bostani and Abdulamir Saiiari studied the physical and mental health of athletes and non-athletes in college students by using random sampling techniques on their research "Comparison Emotional Intelligence and Mental Health between Athletic and Non-Athletic Students", the results showed that there are remarkable differences between athletes and non-athletes, that is to say, regular exercises can promote students' physical and mental health.

Domestic research focused on two aspects: the one concerning the literature on "Zhai" lifestyle, the other concerning the literature on mental health of college students. On the first aspect, Zhou Yi and Wu Hao(2012) argue that there are necessity and rationality for the emergence of "Zhai culture" and the phenomenon can be changed by strengthening ideological education and culture-building based on schools, families and society efforts on their research "what is the fun of home? ---- Analysis college students' house phenomenon" ; Yu GuoNi and Jia Zhi(2012) put forward the similar opinion in their "the 'campus' home behind the phenomenon", they think there are social backgrounds for "Zhai" lifestyle in college campus, and point out the importance to consider higher education management problems; Yang Min(2011) investigated 1100 students of 15 colleges in Shanghai and analyzed their "Zhai" situation, the recognition level of the "Zhai", the degree of "Zhai" life and their opinions on "Zhai" lifestyle in his study "The investigation of the contemporary college student's life", meanwhile, incorporated multiple factors to study the reasons for it.

Aim at the Physical and mental health of students, Xiao Jianwei (2005) select 408 students in Hebei Normal University and Shijiazhuang University as the research object in the book of "Students' Personality, dormitory interpersonal relationships and mental health research". He took Wang Dengfeng's QZPS to measure the popularity of college students in the dormitory. Li Jian (2007) made an assessment from 72 Special evaluation systems and 9 aspects in the "Students Mental Health Evaluation System" according to selecting 3,689 college students across the country as well as Hong Kong, Macao, Taiwan by the questionnaire survey. Liu Yumei (2009) selected 600 students in Tianjin Polytechnic University as the research. She adopt random sampling and cluster sampling method to analysis the Internet influence on college students' physical and mental health, including online behavior, attitude, psychological and physical effects in the "physical and mental health of college students the Internet impact survey".

To sum up the domestic and foreign literature, there has no direct research on the effects that "Zhai" lifestyle has on college students' physical and mental health. So based on survey data, I do quantitative analysis combined with qualitative analysis, on the basis of extensive reading relevant literature, trying to use the theory to extract the data.

\section{The Study Design and Data Sources}

\subsection{Concept Definition}

"Zhai" culture was handed from Japan to China, originating from Japanese "otaku”. With the development of the network, the concept "Zhai" becomes popular increasingly, and it has been a hot societal concern. "Zhai" group in China has been given a new definition: it is an attitude, or a new way of life. These people confine themselves at home, disconnect with the outside world and rely on a variety of technology products. In this paper, "Zhai" refers to those college students who stay in their dormitories all day, rarely go out and seldom communicate with others. They are mainly indicated on two aspects as follows: 1 ) Staying more than 4 hours in the dormitory in the spare time; 2) Getting online more than 3 hours every day.

It is difficult to get a uniform standard because different scholars have different interpretations to the definition of physical health and mental health, so we define unhealthy state based on the standpoints of domestic and foreign scholars as below: 1) Doing not exercise regularly; 2) Being shy about meeting new people, feeling unconfident even inferior; 3) Usually solving problems by repressing one's emotions.

\subsection{Subjects and Methods}

Subject investigated: College students mainly from Sias International University, and the rest of them from other parts of China through random sampling survey of the online questionnaire. They can basically represent the situation of China.

Research methods: Questionnaire survey, SPSS19.0 data processing and analysis system, descriptive statistics, mathematical statistics, and comparative analysis, etc.

\subsection{Data Sources and Indicators to Determine}

From November 2012 to March 2013, the author investigated 310 students who come from Sias International University as well as other colleges of China through questionnaire survey, including 151 males and 159 females 
(48.7\% of male and $51.3 \%$ of female), and the proportion of students from freshman to senior year are 1:3:6:8. 155 students from Sias international college were investigated, including 71 males and 84 females $(45.8 \%$ of male and $54.2 \%$ of female). According to the definition of "Zhai", "Internet time" and "time of staying in their dorms" as classification index are designed in the questionnaire. (The questionnaire see appendix)

\section{The Study Results and Analysis}

\subsection{The Basic Circumstances of "Zhai” Lifestyle Survey}

The author divided students into two categories: "Zhai" and non- "Zhai" according to the definition of "Zhai" in this paper. There are 125 students of "Zhai" life (40.3\% of total number of surveys) and 185 students of non- "Zhai" life (59.7\% of total number of surveys). As a whole, there are majority of students who have healthy lifestyle.

From the point of gender, there are 64 males and 61 females in "Zhai" lifestyle, accounting for $42.4 \%$ and 38.4\% of male and female respondents respectively. From the point of grade, in the "Zhai" type living conditions of the students are 6 freshmen, 11sophomores, 31 junior and 77 senior, representing all grades for $35.3 \%, 21 \%$, 30.4\% and $55.4 \%$ of the total surveyed, see Figure 1. It can be seen, the "Zhai" phenomenon of senior students is the most serious and it occupies a large proportion.

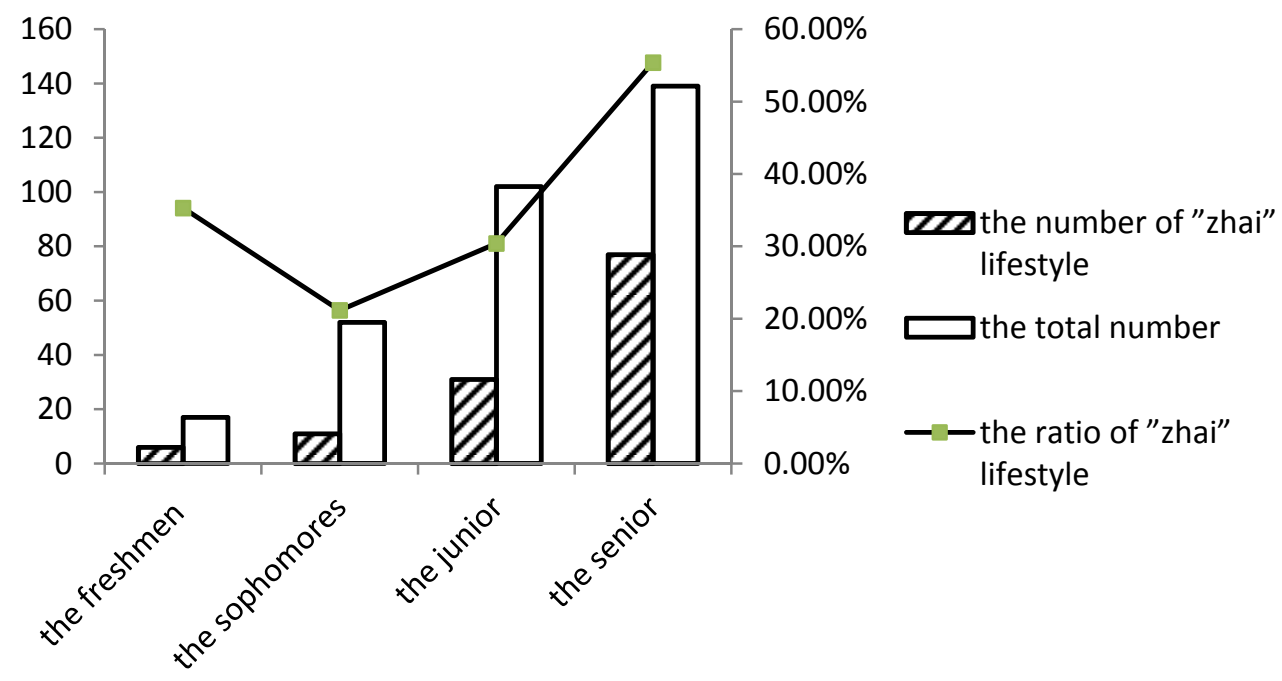

Figure 1: The Different Grade of “Zhai” Lifestyle

\subsection{Spending Too Much Time on Surfing Online at the Dorm}

In the survey, $70 \%$ students would stay at dorm over 4 hours per day, including $20 \%$ of them almost never go out of dorm for a long time .During staying at dorm,46\% students spend from 1 to 3 hours online. What's more, near $20 \%$ of them even let it over 6 hours. Consequently, we have done the correlation analysis on the "staying in the dormitory" and "surfing the Internet every day". The results shows that two variables have positive correlation, the Sig value is zero, less than 0.05 , which can account for spending much time online in dorm. In other words, the more they stay at dorm, the more likely that they surf online, and which also indirectly illustrate the truth that students are not willing to go out with the development of Net, whereas surfing online becomes an obligatory course.

To move the analysis into a deeper way, we've conducted an analysis on the time of students spending on surfing online all grades by ANOVA (Table 1). As a result, the Sig value is 0 , is less than 0.05 , you can come to a conclusion that the time online do exist differences between different grades. Further analysis, by multiple comparison (Table 2), the mainly difference is from senior, who has more time, however, spending on surfing online. The reason is that the graduate students are in a perplex stage, when they are not sure where to go in the future, so they decide to abandoned themselves in visionary world. 
Table 1: One-way Anova Analysis

\begin{tabular}{cccccc}
\hline & Sum of squares & Df & Mean square & F & Sig \\
\hline Between groups & 41.362 & 3 & 13.787 & 23.254 & .000 \\
Within groups & 181.425 & 306 & .593 & & \\
Total & 222.787 & 309 & & & \\
\hline
\end{tabular}

Table 2: The Internet Time for Multiple Comparison

\begin{tabular}{ccccc}
\hline \multirow{2}{*}{ (I) Grade } & (J) Grade & Standard error of mean & Sig \\
\hline \multirow{2}{*}{ Senior } & Freshman & $.522^{*}$ & .198 & .009 \\
& Sophomore & $.820^{*}$ & .125 & .000 \\
& Junior & $.708^{*}$ & .100 & .000 \\
\hline
\end{tabular}

\subsection{Lacking of Doing Exercise}

Students spend much time at the dorm, so they are insufficient of physical excise. In the research (Table 3), we find that doing exercise in their spare time becomes the last one which rare ones namely $1.9 \%$ would prefer. However, when be asked the main arrangement in the leisure, $42.3 \%$ of the students choose to spend time on cellphone or surfing on the Internet. There are merely $14 \%$ of the students will prefer hanging out with friends as the first choice in spare time. As a result staying indoors to surf the Internet occupies a chief part in extracurricular college life. Beyond that, we have learned almost near half of the students who took up $49.4 \%$ spend time online in order to watch movies, videos, TV sets; the students who looked for materials and study occupied on $20 \%$. The amounts of students who cannot let life go on well without network are becoming more and more. In addition, in the survey, there are many schoolmates reflected that they had irregular eating and stomach because they stayed indoors for too long, and some of whom even affected their normal life.

Table 3: The Most Popular Activities in Spare Time

\begin{tabular}{lccc}
\hline The most popular activities in spare time & Frequency & Percent & Cumulative Percent \\
\hline Sleeping & 81 & 26.1 & 26.1 \\
Hanging out with friends & 43 & 13.9 & 40.0 \\
Playing with mobile phone or the Internet & 131 & 42.3 & 82.3 \\
Reading in the library or a classroom & 49 & 15.8 & 98.1 \\
Doing exercise & 6 & 1.9 & 100.0 \\
\hline
\end{tabular}

\subsection{Poor Interpersonal Communication Ability}

Asked if they would feel shy talking to people face to face when compared to online communication, only $25 \%$ students think they have little or no such feeling; reach up to $75 \%$ students have this feeling, even $8 \%$ of them have such problem all the time. Furthermore, asked if they felt difficult to get along with people around, there were $21 \%$ classmates think it is so, most of them think they can better dealing with the relationship in such circumstance. In a word, because of long time never leaving home and lacking of face-to-face communication with people, students' communicative ability is very poor when facing the stranger. But they basically still can get along around the people.

Further analysis between the two variables "feeling shy face to face communication with strangers" and "difficult to get along with people around" of the "Zhai" and non- "Zhai” life state of students. From Table 4 shows, the Sig value is less than 0.05 , refuse to null hypothesis, that is, two kind of way of life of students has significant differences, and "Zhai" lifestyle students are hard to get on well with people and feel shy to communicate with others face to face. The results confirm the curtilage type life on students' negative effects. 
Table 4: Independent Samples Test

\begin{tabular}{|c|c|c|c|c|c|c|}
\hline & \multirow[t]{2}{*}{ Variable } & \multicolumn{2}{|c|}{$\begin{array}{c}\text { Levene’s test for equality } \\
\text { of variances }\end{array}$} & \multicolumn{3}{|c|}{ T-test for equality of means } \\
\hline & & $\mathrm{F}$ & Sig. & $\mathrm{T}$ & Df & Sig. (2-tailed) \\
\hline \multirow{2}{*}{ Shy or no } & Equal variances assumed & 1.004 & .317 & -2.118 & 308 & .035 \\
\hline & Equal variances not assumed & & & -2.106 & 260.876 & .036 \\
\hline \multirow{2}{*}{$\begin{array}{c}\text { Difficult to get } \\
\text { on with people } \\
\text { or not }\end{array}$} & Equal variances assumed & 2.716 & .100 & -2.584 & 308 & .010 \\
\hline & Equal variances not assumed & & & -2.579 & 264.499 & .010 \\
\hline
\end{tabular}

\subsection{Confidence Is Not Enough}

Confidence represents everyone for his trust and it is a positive attitude toward life. Once Seting up confidence, thought will become optimistic, open-minded, and life will become better, so the degree of students' self-confidence directly reflects the life attitude. In the survey (Table 5), less than $25 \%$ of students think they are confident, most of the rest think they are not confident, even don't know. It shows that the present students lack a positive life state. In the correlation analysis of the degree of confidence and feeling shy, there is a negative relationship between the two variables, significant Sig Value of 0.008 , less than 0.05 , which is statistically significant. The results indicate that students' confidence is not enough, which leads to the students' shyness in the face of the strangers.

Table 5: The Degree of Confidence

\begin{tabular}{lccc}
\hline The degree of confidence & Frequcncy & Percent & Cumulative Percent \\
\hline Very confident & 6 & 1.9 & 1.9 \\
Confident & 67 & 21.6 & 23.5 \\
Sometimes not too confident & 197 & 63.5 & 87.1 \\
Not confident, often feel inferiority, & 32 & 10.3 & 97.4 \\
Don't know & 8 & 2.6 & 100.0 \\
\hline
\end{tabular}

\subsection{Students Can Regulate Themselves}

A person's physical and mental health harmony or not, the key is to adjust itself. In psychology, self-adjusting is defined self-control over their emotions and behaviors. In simple words, their own state of mind will not change because of the external environment changes, etc. Psychology also defines many adjustment methods, of which the changing and abreacting are kinds of active process, and depression is negative, of course the natural adjustment is sometimes solution of escaping.

According to the survey, in the face of a bad mood on the handling of nearly 70\% students chose the positive transfer or vent, and relatively negative approach accounts for the proportion of less. This indicates that Students can regulate themselves. However, further analysis between the two variables "Depress" and "Others" of the "Zhai" and non"Zhai" life state of students. The results shows, all the Sig value is less than 0.05, refuse to null hypothesis, that is, two kind of way of life of students has significant differences, and "Zhai" lifestyle students are more likely to be depressed when the mood is bad.

\subsection{The Cognitive on "Zhai"}

In this survey, different respondents have different views on the phenomenon of "Zhai”. Only 36.5\% think that "Zhai" is bad and negative attitude to life; another $63.5 \%$ think that it is out of their business; even $7.7 \%$ believe that "Zhai" is a good way to life reflecting with freedom life attitude.

In addition, In the research, we find that the reason why many students become "Zhai", 74.5\% think that "they have nothing to do", "addicted to online games", "have no opportunity to go out"; $16.8 \%$ students think that different people have different choice, it is not a must be choice; $9 \%$ of the students think the reason is they do not want to communicate with people and they just want to stay away from society. We can reach a conclusion, it is not that they do not want to communicate with people and they just want to stay away from society, but some objective reasons which make students have no aim. 


\section{Discussion}

Known from the study results and analysis of the above, we now discuss them as follows:

Firstly: The results show that most of the students have healthy lifestyle, but there also are quite a lot of students staying at home for a long time. From the point of gender, the male students obsessed to "Zhai" lifestyle are slightly more than that of female. Combining the survey about college students' Internet content, this phenomenon may result from males more likely to indulge in network and games. From the point of grade, the "Zhai" phenomenon of senior students is the most serious and it occupies a large proportion. The reason for this situation may be that the seniors are in the transition period of learning and employment, they are dazed about the future, and they have some difficulties in taking postgraduate entrance exams or finding a job, so they choose home in the bedroom or a temporary escape.

Secondly: The results prove although "Zhai" has enriched the culture diversity to some degree, it may produce plenty of negative influence on college students' physical and mental health. As mentioned above, in spare time, Students spend much time in enjoying computers and playing with their cellphones, and few students doing physical exercise. If things go on like this, their physical fitness will decline. Once they come across emergency things, they will feel exhausted. Besides, their eyesight will also go down during their enjoying computers and cellphones. To move the results into a deeper way, the more they stay at dorm, the more likely that they surf online, which leads to less face-to-face communication with others. Such vicious circle, will accentuate even more the health declines. In reaction to the phenomenon, as students we should improve our self-control, learn to resist the temptation of the Internet, and make full use of the Internet to benefit study. The school should show the disadvantage of addicting to online games; let them take warning from these things. Also, the school must be sure to give the right guidance to students.

Thirdly: The results indicate "Zhai" lifestyle students are hard to get on well with people and feel shy to communicate with others face to face. They have poor interpersonal communication ability, their self-confidence is insufficient, their characters are very lonely, and they are more likely to be depressed when the mood is bad. In a word, the results confirm the curtilage type life on students' negative effects. As college students themselves should be aware it. And as the school should strengthen the guidance and the education of students, and make them set up the correct outlook on life.

Afterwards: According to the results of the analysis above, we have learned the "Zhai" phenomenon of senior students is the most serious, and they have more time, however, spending on surfing online instead of doing exercise. That especially justifies “Zhai” lifestyle has the great negative effect on the graduates' body and mind. The reason is that they are in a perplex stage, when they are not sure where to go in the future, so they decide to abandoned themselves in visionary world. Confronted with this problem, college should take their responsibilities to do more guidance, and concentrate on the graduate students' mental change all the time. Furthermore, college should give them positive life directions and suggestions. Last but not least, students as adults who are going to enter the real society ought to solve the problem by themselves to make future plans in advance.

Finally: Since "Zhai” lifestyle has the great negative effect on the college students' physical and mental health, then why there are so many students choose it? A very important reason is they have not aware of the harm of "Zhai". As the results mentioned above, only 36.5\% students think that "Zhai" is bad and negative attitude to life. Even there are quite a number of students accepted the "Zhai". In view of this reality, as the school should guide students to correctly think of "Zhai", and make them understand the negative effects much more than the positive effects. As a student, we should rationally look upon this problem and never drift with the current, only understanding the correct way of life, can we adapt to the development in the future.

\section{The Conclusion and Rationalization Proposal}

\subsection{Conclusion}

According to the above data analysis and discussion can get the following conclusions: 1) More and more college students (especially male and senior students) choose "Zhai" lifestyle; 2) The results confirm "Zhai" produces plenty of negative influence on college students' physical health. Because of lack of doing exercise, their physical fitness drops. They are more curtilage in the dormitory surfing the Internet. Such vicious circle, will accentuate even more the health declines; 3) The results indicate "Zhai" has affected college students' mental health. "Zhai" lifestyle students are hard to get on well with people and feel shy to communicate with others face to face. They have poor interpersonal communication ability. Their self-confidence is insufficient, and they are more likely to be depressed 
when the mood is bad; 4) Although "Zhai" lifestyle has the great negative effect on health, college students have not aware of the harm of "Zhai". Even quite a number of students accepted the "Zhai".

\subsection{Rationalization Proposal}

Campus life is different from the students' family environment, and it is the collectivization and the institutional surroundings. On the one hand, the students need to face the four years of studies, and to deal with the interpersonal relationship and employment pressure. On the other hand, due to the lack of constraints from parents and teachers, the students have their own free space. In such a special environment, it is easy to cause "Zhai" type way of life, which will affect students' physical and mental health. Further, lacking of doing exercise, physical quality will drop. To get to the Internet to kill time, or even skip classes for it, which gradually form the negative lazy attitude. Once forming this atmosphere throughout the campus, it is not only a person's question, but also affect the future development of generation, so the phenomenon must be correctly guided. The following is relevant policy recommendations.

\subsubsection{Their Own Level}

Students should learn the positive and optimistic attitude to solve the problem. As the hope of the future society, students should set up the lofty ideal, the correct outlook on life and values, and make every effort with down-to-earth. Meanwhile, they shouldn't have the negative attitude towards life, the more can't let the noise of others' negative lifestyle affect themselves.

\subsubsection{The Family Level}

Family plays an important role on the growth of the individual. Although the students have left the parents to live independently, the influence of the family is still ubiquitous .Parents should pay more attention to their psychological state, and help them to cultivate a positive attitude towards life.

\subsubsection{The School Level}

University is the place where students live and learn, and its impact on the students is the most direct. Responsibility of university is not only teaching, but also helping students set up the correct outlook on life. Therefore, first of all, implementing the future planning of college students, and you can set up related lectures, making them genuinely realize its importance; Second, Schools should teach students to reasonable use of leisure time, and encourage the students to take an active part in interpersonal communication; Finally, for those students of serious curtilage type living conditions, we should give more positive guidance and help.

\subsubsection{The Society Level}

College students' curtilage type way of life should deserve special attention by the whole society. After all, students' stage of life is special. As a society, we should give them correct guidance, but not blindly criticize; we should learn to understand them and help them. In a word, we must face up to the "Zhai" phenomenon of college students, and try our best to help students under the joint efforts of the whole society.

\section{References}

Mehdi Bostania \& Abdulamir Saiiarib. (2011). Comparison emotional intelligence and mental health between athletic and non-athletic students. Procedia - Social and Behavioral Sciences, 27(9), 100-104.

Balasubramanian Madhan. (2009). Mental health of postgraduate orthodontic students in India: a multi-institution. International Dental Education Survey, 30(7), 24-27.

Farideh Bastani \& Sara Hashemi. (2012). Effects of a web based lifestyle education on general health and severity of the symptoms of Premenstrual Syndrome (PMS) among female students: a randomized controlled trial. Procedia-Social and Behavioral Sciences, 46(9), 57-60.

Wu Qing. (2011). The China youth network public participates in the essence and the historical development of enlightenment. China Youth Study, 11(3), 12-15.

Wang Quanan. (2012). Curtilage phenomena analyses. Journal of Changchun Education Institute, 28(6), 35-37.

Yu You. (2011). College students curtilage phenomenon research. China Education Innovation Herald, 30(4), 72-76.

Li Lulu. (2009). The college student, you home? - walked into the university campus housing culture today. Journal of Renmin University of China, 23(3), 54-57. 
Guo Shaodong, \& Chai Shujian. (2012). The investigation of the phenomenon of college students staying in. Journal of Yangzhou University, 15(3), 44-48.

Liu Yuanyuan, \& Wang Yuanyuan. (2008). Analyses of several related problems on college students' otaku. The Wealth of Networks, 20(5), 98-102.

\section{Appendix}

\section{Questionnaire of “Zhai” Lifestyle's Effects on College Students' Physical and Mental Health}

Dear friends, thanks for your help. I'm doing a paper about “Zhai” lifestyle's effects on college students' physical and mental health. This questionnaire survey is below. Your answer will be of great help to me, and the data and information which you filled will not be disclosed. Would you please truthfully answer, thank you for your cooperation.

1).What is your gender? ( )
A. Male
B. Female

2). What' s your grade? ( )
A. Grade one
B. Grade two
C. Grade three
D. Grade Four

3). What kind of way that you usually tend to to contact friends? (MCQ). ( )
A. Meet
B. Call up
C. Send message
D. Blog, etc.
E. E-mail

F. QQ, fetion, micro-channel, MSN, Skype, etc. G. Write a letter

4). What do you do in your spare time (except sleeping)? Primary( );secondary( );finally( )
A. Sleep
B. Hang out with friends
C. Play with mobile phone or the Internet
D. Read in the library or a classroom
E. Do exercise

5). How long do you stay in the dormitory one day on average? ( )
A. 1-3 hours
B. 4-5 hours
C. 6-10 hours
D. Rarely go out

6). How long do you surf the Internet every day? ( )
A. Within 1 hour
B. 1-3 hours
C. 3-6 hours
D. More than 6 hours

7). What do you often do on the Internet? ( $\quad$ )
A. Look for material to learn
B. Flip through news
C. Play games
D. Watch videos /movies/TV sets
E .Chatting
F. Accept /Send E-mails
G. Shopping online

8). If you feel shy talking to people face to face when comparing to online communication? ( )
A. Yes
B. Most of the time
C. Sometimes
D. Rarely
E. Don’t feel so

9). How do you normally handle, when you encounter unhappy or depressed? ( )
A. Change(eat or entertainment)
B. Abreact(cry, pour out, do exercise, keep a diary)
C. Depress(drink, sleep)
D. Natural adjustment

10). Do you find it difficult to get along with people around? ( )
A. Often
B. Sometimes
C. Occasionally
D. Rarely
E. Never

11). If you will feel disoriented, when you have a lot of free time? ( )
A. Never
B. Sometimes
C. Most of the time
D. Always

12). Do you feel confident? ( )
A. Very confident
B. Confident
C. Sometimes not too confident
D. Not confident, often feel inferiority
E. Don't know

13). What do you think of the reason that some college students curtilage in the dormitory? ( )
A. Hobbies and interests
B. Have no opportunity to go out
C. Addicted to online games
D. Have nothing to do 
E. Don't want to communicate with people F. Escape society

14). What do you think of the college students' “Zhai” phenomenon? ( )

A. Nothing, just a way of life $\quad$ B. Bad, negative attitude towards life $\quad$ C. Very good, very casual D. No ideal 